$+\mathrm{CD} 3+$ cells is significantly reduced from heterozygous to knockout animals following injury.

Conflict of interest None

\section{BS32 UNTARGETED METABOLOMICS INTERROGATION OF ADIPOSE TISSUE SECRETOME FROM PARTICIPANTS OF THE OXFORD COHORT FOR HEART, VESSELS \& FAT HIGHLIGHTED CERAMIDES AS POTENTIAL ADIPOKINES MODULATING VASCULAR REDOX SIGNALLING IN CARDIOVASCULAR DISEASE}

${ }^{1}$ Nadia Akawi ${ }^{*},{ }^{2}$ Antonio Checa, ${ }^{1}$ Christos Kotanidis, ${ }^{1}$ loannis Akoumianakis, ${ }^{5}$ Evangelia Daskalakis, ${ }^{6}$ Craig Wheelock, ${ }^{7}$ Charalambos Antoniades. ${ }^{1}$ Oxford University; ${ }^{2}$ Karolinska Institute

\subsection{6/heartjnl-2019-BCS.195}

Background Hypothesis-free unbiased -omics approaches have started to revolutionise our understanding of adipose tissue (AT) biology providing an exciting opportunity for the discovery of yet unknown adipokines that drive the currently recognised cross-talk between the AT and the vasculature. Methods: Untargeted metabolic profiling of the secretome of paired thoracic and subcutaneous AT samples from 48 patients undergoing cardiac surgery (OxHVF Study) along with subsequent sphingolipids-targeted quantification were performed using liquidchromatography mass-spectrometry. Immortalised human aortic endothelial cells (teloHAEC) and human umbilical vein endothelial cells (EA.hy926) were treated exogenously with ceramides for $20 \mathrm{~min}$ and superoxide (O2.-) was measured using lucigeninenhanced chemiluminescence. Results: Metabolomics differential and enrichment analyses highlighted the significant differences in sphingolipids secretion levels between the two fat depots. Higher amount of long and very-long chains ceramides were produced and secreted from the thoracic depot with C16-ceramide representing the most abundant differentially secreted ceramide (figure 1). Compared to the lowest tertile, the middle and highest tertiles of ceramides of thoracic AT were significantly associated with enhanced O2.- production in patients' vessels (figure 2-A). ThAT and ScAT highest and middle tertiles of ceramides were found to be associated with reduced endothelium-dependent vascular relaxation in response to acetylcholine, a fundamental feature of endothelial dysfunction (figure 2-B). Exogenous treatment of teloHAEC and EA.hy926 with ceramide increased basal O2.- and endothelial nitric oxide synthase (eNOS) uncoupling evidenced by more negative O2.- after addition of LNAME, an inhibitor of eNOS. Moreover, NADPH-stimulated O2.- generation and Vas2870-inhibitable O2.- were significantly higher in cells pre-treated with ceramide compared to untreated counterparts. Conclusions: In this study, we demonstrate for the first time that sphingolipids, in particular ceramides, secreted from the AT of cardiac patients modulate their vascular redox state and vasomotor tones, the key determinants of dysfunctional endothelium-a hallmark of cardiovascular disease.

Conflict of interest None
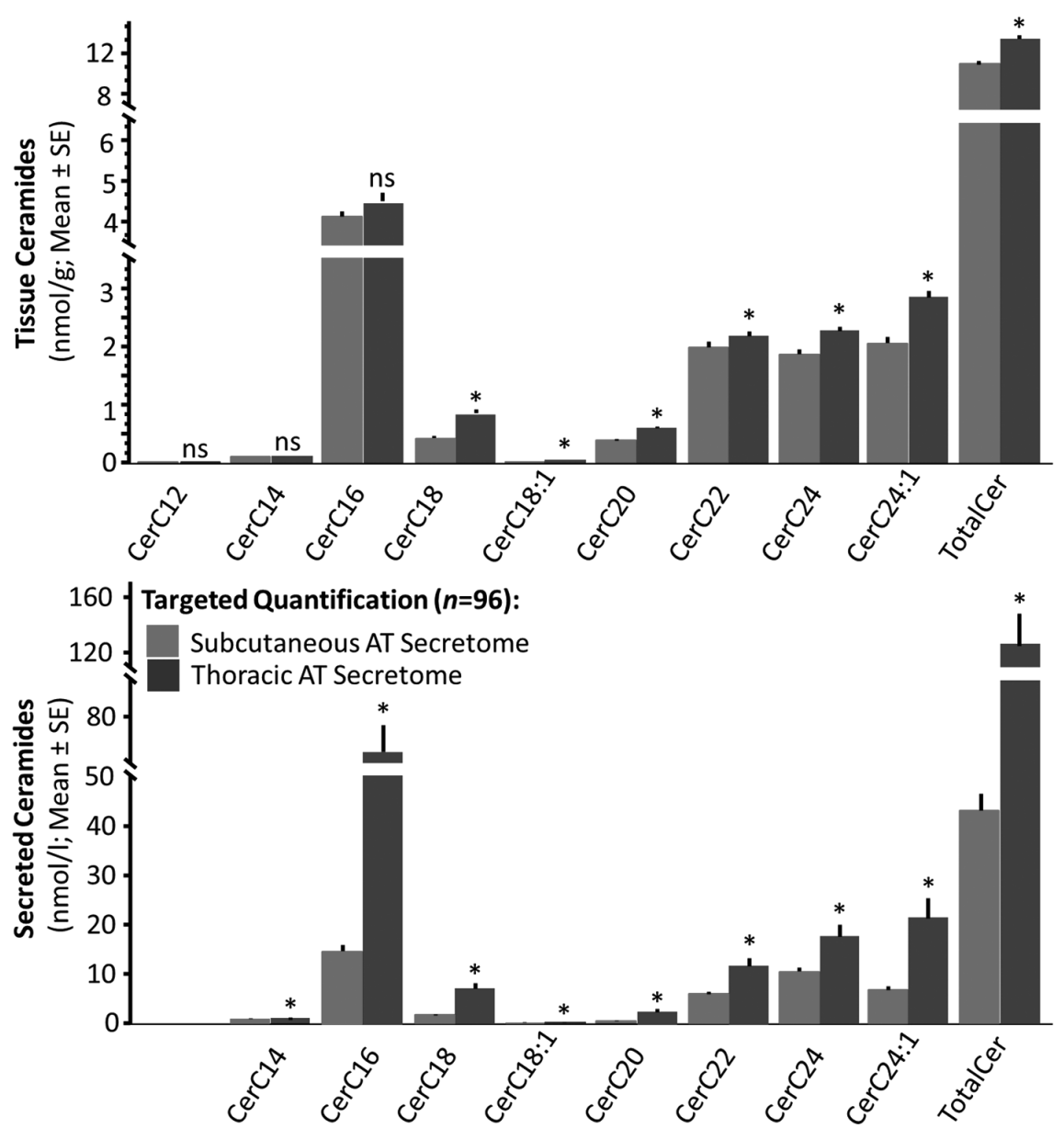

Abstract BS32 Figure 1 